\title{
Comparación de la eficacia anestésica pulpar por medio de las técnica incisiva y bloqueo del nervio alveolar inferior
}

\author{
Rodríguez-Hernández Carlos*, Hernández-Nájera Rafael**, Pozos-Guillén Amaury de Jesús**, \\ Reyes-Vela Enrique ${ }^{* * * *}$, Masuoka-Ito David ${ }^{* * * *}$, Gaitán-Fonseca Cesar Iván ${ }^{* * * * *}$.
}

\footnotetext{
- Resumen

- Introducción: El manejo y control del dolor representa un factor cla-

- ve en el desarrollo de la práctica odontológica. Objetivo: Comparar

- el grado de anestesia pulpar alcanzado en dientes antero-inferiores

- utilizando la técnica del nervio incisivo y el bloqueo del nervio alveolar

- inferior. Materiales y métodos: Un ensayo clínico controlado fue rea-

- lizado donde se evaluaron 33 pacientes sometidos a una valoración

- pre-anestésica con la prueba de sensibilidad al frio para verificar el

- estado pulpar. Posteriormente se infiltró $1.8 \mathrm{~mL}$ de lidocaínalepinefri-

- na I:100 000 con un periodo de latencia de cinco minutos. Finalizado

- este periodo se realizó nuevamente valoración del estado pulpar con

- la prueba de sensibilidad al frio. Resultados: Los resultados obtenidos

- fueron contabilizados con un cronómetro (///00). Para el grupo de

- la técnica del bloqueo del nervio alveolar inferior se registró una res-

- puesta pulpar inicial de $3.38 \pm 1.05$ s y una respuesta final de 1.80

- $\pm 1,87 \mathrm{~s}$. La técnica del nervio incisivo reportó una respuesta inicial

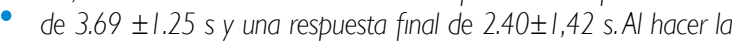

- comparación por grupos se encontró una diferencia estadísticamente

- significativa $\left(p<0.001, X^{2}\right)$. Conclusiones: La técnica del bloqueo del

- nervio alveolar inferior mostró mayor eficacia en anestesia pulpar que

- la técnica del nervio incisivo. LUX MÉDICA AÑO 8, NÚMERO 25, SEPTIEM-

- BRE-DICIEMBRE 2013, PP 23-28
}

Palabras clave: Anestesia pulpar, nervio incisivo, nervio

$\bullet$ alveolar inferior.

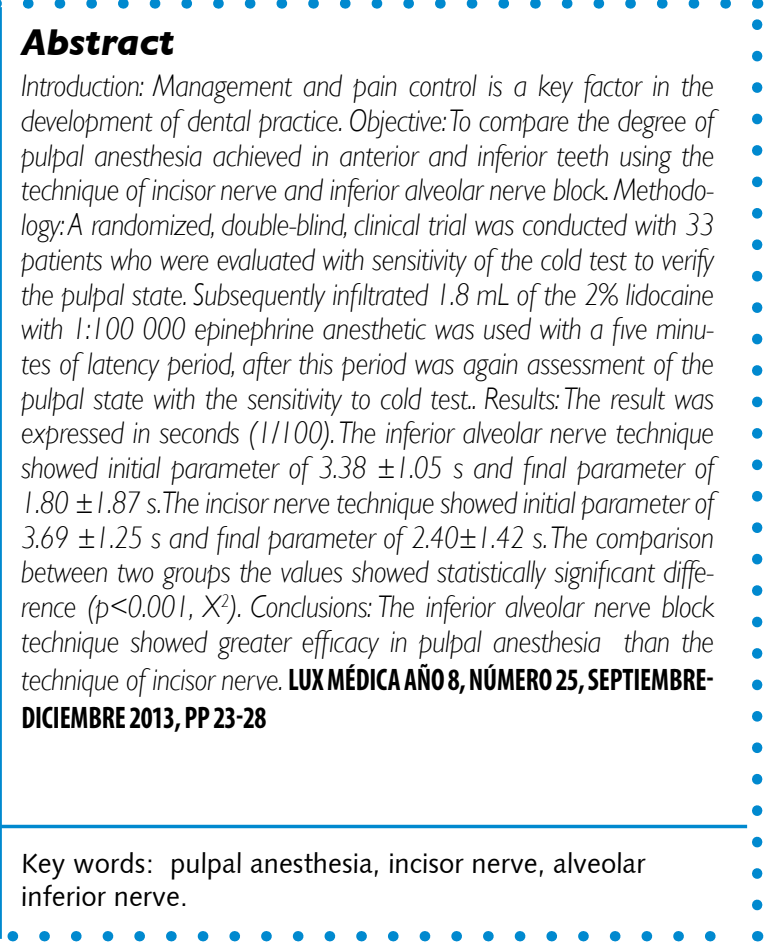

\section{Abstract} pulpal anesthesia achieved in anterior and inferior teeth using the echnique of incisor nerve and inferior alveolar nerve block. Methodopatients who were evaluated with sensitivity of the cold test to verify the pulpal state. Subsequently infiltrated $1.8 \mathrm{~mL}$ of the $2 \%$ lidocaine with 1:100 000 epinephrine anesthetic was used with a five minutes of latency period, after this period was again assessment of the pulat state with the sensitivity to cold test.. Results: The result was $1.80 \pm 1.87$ s. The incisor 1.38 technique showed intial parameter of $3.69 \pm 1.25 \mathrm{~s}$ and final parameter of $2.40 \pm 1.42 \mathrm{~s}$. The comparison between two groups the values showed statistically significant diffeechnique of incisor nerve IUXMÉDICA AÑ 0 8, NÚMER025, SEPTIEMBREDICIEMBRE 2013, PP 23-28

* Especialista maxilofacial. Departamento de Estomatología, Centro Ciencias de la Salud, Universidad Autónoma de Aguascalientes.

** Residente de segundo año de la Maestría de Ciencias Biomédicas Área Rehabilitación Bucal, Universidad Autónoma de Aguascalientes

*** Doctor en Ciencias Biomédicas Básicas, Facultad de Estomatología, Universidad Autónoma de San Luis Potosí.

**** Maestro en Ciencias Odontológicas, Departamento de Estomatología, Centro Ciencias de la Salud, Universidad Autónoma de Aguascalientes.

***** Doctor en filosofía, Departamento de Estomatología, Centro Ciencias de la Salud, Universidad Autónoma de Aguascalientes.

******Maestro en Endodoncia, Departamento de Estomatología, Centro Ciencias de la Salud, Universidad Autónoma de Aguascalientes

Fecha de recibido: 17 de mayo 2013

Fecha de aceptación: 9 de octubre 2013

Correspondencia: Carlos Rodríguez Hernández. Departamento de Estomatología del Centro de Ciencias de la Salud. Universidad Autónoma de Aguascalientes. Avenida Universidad \#940 Ciudad Universitaria. CP 20131. Aguascalientes, Ags., México. Teléfono 9108430.Correo electrónico carlosmaxillo@ yahoo.com.mx 


\section{Introducción}

El manejo y control del dolor representa un factor clave en el desarrollo de la práctica odontológica. ${ }^{1,2}$ Estudios previos reportados han evaluado la efectividad de los diferentes tipos de anestésicos locales y técnicas clínicas descritas en diversos procedimientos odontológicos. ${ }^{3,4}$ Todos los anestésicos locales presentan eficacia en odontología, la variabilidad de la misma se presenta en la técnica empleada y el conocimiento de puntos anatómicos por parte del operador. La duración del efecto anestésico depende del tiempo en que la droga está en contacto con el nervio bloqueando los canales de sodio. ${ }^{5-8}$ El bloqueo del nervio alveolar inferior (BNAI) es la infiltración con más frecuencia empleada para anestesia local en procedimientos odontológicos, sin embargo tiene reportes de un rango de fracaso que oscila entre $7-75 \%$. Esta técnica cuando es colocada adecuadamente proporciona efectos anestésicos importantes inclusive a nivel pulpar. ${ }^{9-10}$ Malamed reporta que con la técnica del bloqueo del nervio incisivo, una variante del bloqueo mentoniano, se puede lograr un grado importante de anestesia pulpar permitiendo realizar cualquier procedimiento sin presencia de dolor. ${ }^{11}$

La literatura reportada acerca de esta técnica incisiva es limitada, la técnica es descrita por Malamed donde menciona que se recomienda utilizar aguja dental corta 25G o 27G, ubicando como punto de infiltración, el pliegue mucobucal a la altura del foramen mentoniano o anterior al mismo. Los puntos de referencia que deben ser considerados son los premolares mandibulares y el pliegue mucobucal teniendo cuidado de orientar el bisel de la aguja hacia el hueso. De frente al paciente y con éste en posición decúbito o semidecúbito, se pide al paciente que cierre ligeramente su boca y se localiza el foramen mentoniano traccionando el labio y la mucosa bucal, se coloca el dedo índice a la altura del primer molar inferior y se desplaza en sentido anterior hasta ubicar la concavidad formada por la emergencia del nervio mentoniano. Se prepara el tejido con aplicación de anestésico tópico por lo menos durante un minuto y se procede a puncionar manteniendo los tejidos tensos, a la altura del canino o primer premolar y dirigiendo la jeringa hacia distal, profundizando 5-6 $\mathrm{mm}$ (no es necesario ingresar al foramen mentoniano). Se aspira y si resulta negativa, se infiltra un tercio del cartucho en un lapso aproximado de 20 segundos. ${ }^{12,14}$ Esta técnica hace énfasis en la necesidad de presionar extra o intraoralmente el sitio de infiltración durante la latencia, para asegurar el ingreso del anestésico al canal mentoniano y su contacto con el nervio incisivo obteniendo así anestesia a nivel pulpar. ${ }^{12,14}$ El objetivo del presente estudio es la evaluación del grado de anestesia pulpar logrado en dientes antero-inferiores utilizando la técnica de bloqueo del nervio incisivo y el bloqueo del nervio alveolar inferior (BNAI). 


\section{Materiales y Métodos}

Se desarrolló un ensayo clínico controlado con 33 pacientes entre 20-23 años de edad, que pertenecían al quinto semestre de la licenciatura de médico estomatólogo del Departamento de Estomatología de la Universidad Autónoma de Aguascalientes, a quienes se les realizó historia clínica y firma de consentimiento informado para su participación en este estudio. Todos los pacientes en su evaluación médica y odontológica estaban ausentes de cualquier enfermedad sistémica y dental en las piezas seleccionadas. Los pacientes fueron distribuidos aleatoriamente y asignados a cada grupo de estudio. El grupo de estudio 1 correspondió a los pacientes sometidos al bloqueo del nervio incisivo y el grupo de estudio 2 correspondió a los sometidos al bloqueo del nervio dental inferior, para la aplicación de las técnicas anestésicas.

\section{La metodología desarrollada se dividió en cuatro fases:}

1.- Valoración pre-anestésica

2.- Bloqueo del nervio incisivo

3.- Bloqueo del nervio dental inferior (BNAI)

4.- Valoración post-anestésica

Para las fases de valoración pre-anestésica y post-anestésica los órganos dentales fueron evaluados por un especialista en endodoncia realizando la prueba al frio con Endo Ice ${ }^{\circledR}$ (diclorodifluorometano). La prueba al frio es una de las que se aplica con mayor frecuencia a nivel clínico, básicamente consiste en colocar un estímulo frío sobre el diente a evaluar. Existen varias opciones para desarrollar esta prueba entre las que se pueden mencionar: hielo, agua fría, dióxido de carbono, cloruro de etilo y diclorodifluorometano ${ }^{15}$. Para la realización de la prueba se requiere del consentimiento del paciente, posteriormente se realiza aislamiento relativo sobre el diente a evaluar y un diente control, si se presenta la respuesta al estimulo frío, se contabiliza en segundos (con un cronómetro) y registra para hacer el diagnóstico clínico. Las fases del bloqueo del nervio incisivo y nervio dental inferior fueron realizadas por un mismo operador especialista en cirugía maxilofacial.

\section{Fase 1: Valoración pre-anestésica}

Para realizar la determinación del estado y vitalidad pulpar de los órganos anteroinferiores a evaluar, se realizó la prueba de vitalidad pulpar de frío con la solución Endo Ice ${ }^{\circledR}$, previa colocación del anestésico15. Se contabilizó y registró la respuesta pulpar al estímulo frío en segundos por medio de un cronómetro (1/100).

\section{Fase 2: Bloqueo del nervio incisivo}

Antes de la colocación de la técnica anestésica se aplicó anestésico tópico con cloruro de lidocaína al 10\% por intervalo de dos minutos en el sitio donde se colocó la punción. La técnica anestésica del bloqueo incisivo fue desarrollada de acuerdo con lo descrito por Malamed utilizando $1.8 \mathrm{~mL}$ de lidocaína/epinefrina 1:100 000 abarcando la zona de los órganos dentales 31, 32 y 33 (nomenclatura de la Federación Internacional Dental, FDI). Se determinó un periodo de latencia de cinco minutos antes de realizar la valoración post-anestésica.

\section{Fase 3: Bloqueo del nervio dental inferior}

Previa colocación de la punción se aplicó anestesia tópica con cloruro de lidocaína al $10 \%$ por un tiempo de dos minutos. La técnica del bloqueo del nervio dental inferior fue colocada según la literatura empleando $1.8 \mathrm{~mL}$ de lidocaína/epinefrina 1: 100,000 considerando los órganos dentales 41, 42 y 43 (nomenclatura FDI) para la valoración post-anestésica (Figura 1). Se determinó un periodo de latencia de 5 minutos para esta valoración. Esta técnica fue empleada un día después de haber aplicado la técnica incisiva.

\section{Fase 4: Valoración post-anestésica}

Después de la infiltración anestésica en la zona de acuerdo con la técnica aplicada y transcurrido el tiempo de cinco minutos de 
latencia, se procedió a realizar la valoración post-anestésica de la zona a evaluar. La valoración se realizó nuevamente con la prueba de vitalidad de frío Endo Ice ${ }^{\circledR}$ en ambos grupos experimentales, para evaluar el grado de anestesia pulpar contabilizando el tiempo de respuesta en segundos por medio de un cronómetro (1/100).
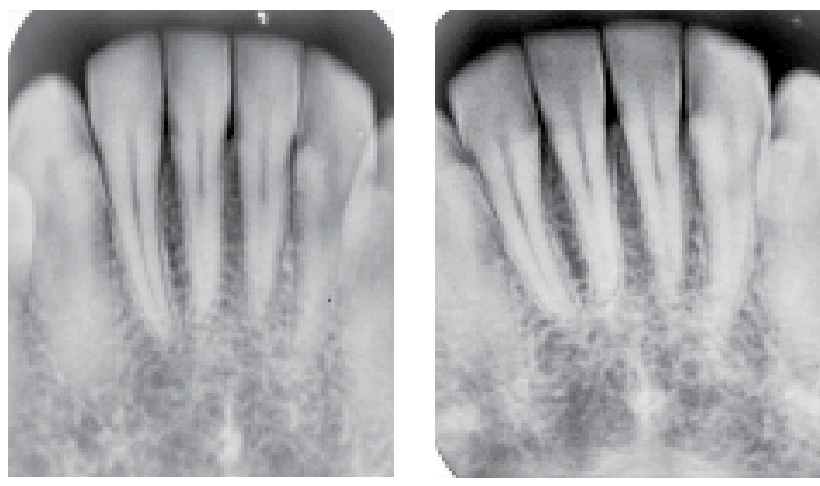

Figura 1. Órganos dentales $(31,32,33,41,42,43)$ evaluadas en las fases pre y post anestésica en la prueba de vitalidad pulpar.

\section{Resultados}

Los resultados se analizaron por técnica anestésica obteniendo promedios, desviación estándar y media de cada uno de los grupos de estudio. En la tabla 1 se observan los resultados obtenidos en el grupo de la técnica del bloqueo incisivo en el momento pre y post anestesia.

\section{Tabla I}

\section{Respuesta pre y postanestésica en pacientes, utilizando la técnica del bloqueo incisivo}

\begin{tabular}{|ccc|}
\hline $\mathbf{n}=\mathbf{6 6}$ & Promedio $\pm \mathbf{D E}$ & Media (Rango) \\
Respuesta post-anestesia (segundos) $(\mathrm{n}=66)$ & $2.40 \pm 1.42$ & $2(0-9)$ \\
\hline Respuesta pre-anestesia (segundos) $(n=66)$ & $3.69 \pm 1.25$ & $3(2-8)$ \\
\hline
\end{tabular}

De acuerdo con los resultados obtenidos en la tabla 1 se puedo observar una disminución de la respuesta inicial pulpar posterior a la aplicación de la técnica anes- tésica. En la tabla 2 se observan los resultados obtenidos al momento de valorar la respuesta de la vitalidad pulpar en la etapa pre y post anestésica.

\section{Tabla 2}

\section{Respuesta pre y postanestésica en pacientes, utilizando la técnica del bloqueo dentario inferior}

\begin{tabular}{|ccc|}
\hline $\mathbf{n}=\mathbf{6 6}$ & Promedio $\pm \mathbf{D E}$ & Media (Rango) \\
Respuesta post-anestesia (segundos) $(\mathrm{n}=66)$ & $1.80 \pm 1.87$ & $2(0-8)$ \\
\hline Respuesta pre-anestesia (segundos) $(n=66)$ & $3.38 \pm 1.05$ & $3(2-7)$ \\
\hline
\end{tabular}


De acuerdo con los resultados obtenidos en esta técnica anestésica se pudo determinar una disminución en la respuesta pulpar inicial posterior a la aplicación de la solución anestésica.

Al realizar una comparación entre grupos de estudio para valorar la disminución de la respuesta de la vitalidad pulpar después de aplicar cada una de las técnicas anestésicas se logró determinar el origen de las diferencias entre los grupos, encontrándose una diferencia estadísticamente significativa $(p<0.001)$ mostrado en la figura 2.

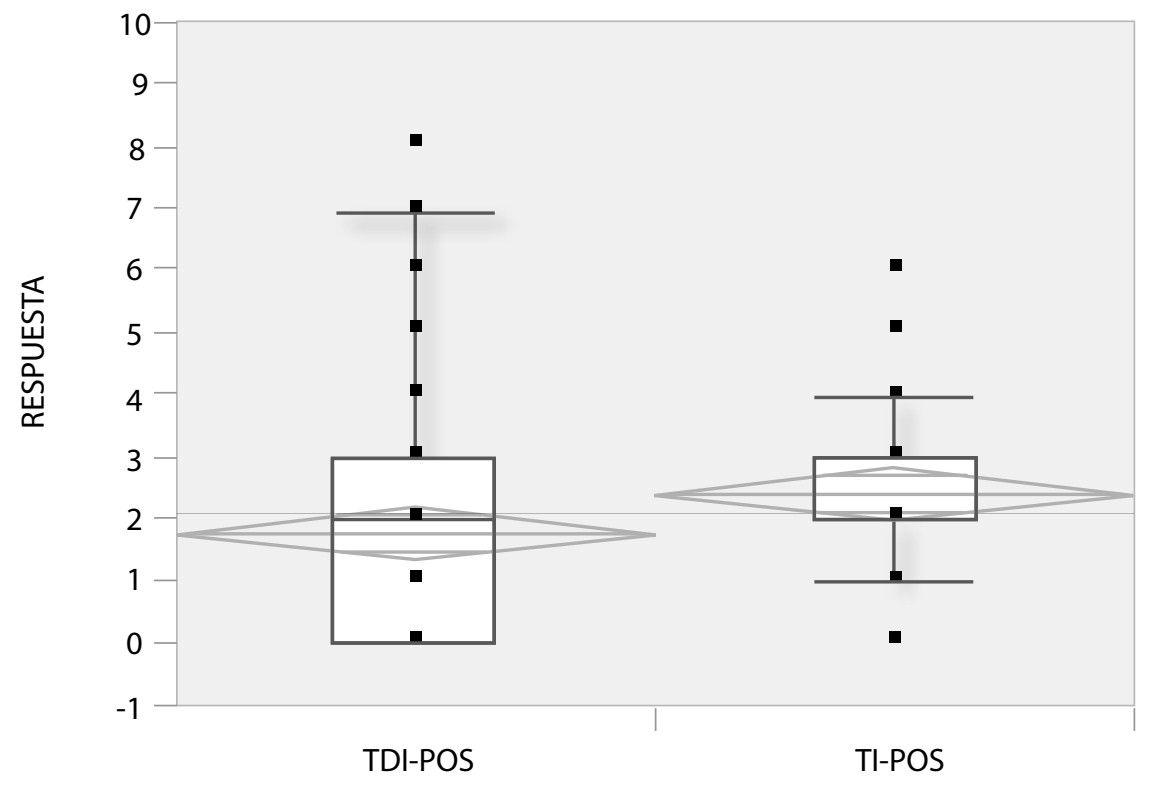

TRATAMIENTO

Figura 2. Comparación entre grupos de estudio de las técnicas anestésicas.

\section{Discusión}

La técnica incisiva en lo reportado por Malamed puede proporcionar efectos importantes a nivel pulpar ya que al realizar la técnica, como parte final de la misma se realiza una presión extra o intraoralmente en el sitio de infiltración durante la latencia, para asegurar el ingreso del anestésico al canal mentoniano y su contacto con el nervio incisivo obteniendo así anestesia a nivel pulpar. Dentro de las indicaciones para esta técnica se pueden realizar procedimientos odontológicos como exodoncia, preparacio- nes o tallados protésicos, colocación de implantes en la zona de premolares, raspados y alisados radiculares, cirugías periodontales y tratamientos de endodoncia. ${ }^{14}$

La técnica del bloqueo del nervio dentario inferior permite anestesiar la rama del mismo nombre, además de lograr anestesia en la rama lingual y bucal. Es el bloqueo más utilizado en odontología pero reporta un fracaso importante al momento de su aplicación, principalmente por una falla en la técnica por parte del operador. Algunos otros factores por lo que esta técnica puede 
llegar a fracasar puede deberse a la presencia de alteraciones anatómicas (presencia de agujeros accesorios, nervio dentario y canales mandibulares bífidos). Otros factores que pueden contribuir al fracaso se deben al abuso de drogas, alcoholismo o trastornos fisiológicos. Dentro de las indicaciones para esta técnica se pueden realizar prácticamente la mayoría de los procedimientos odontológicos, debido a que esta técnica permite anestesiar de manera profunda mucosa, hueso, periostio y la pulpa de molares, premolares e incisivos mandibulares, además de la encía, piel, mucosa del labio inferior y la piel del mentón. ${ }^{14}$
De acuerdo con los resultados de este estudio se logró determinar que la técnica del bloqueo del nervio dentario inferior presentó mayor eficacia en comparación con la técnica mentoniana, además de observar en la mayoría de las mediciones de la anestesia pulpar, en la técnica mentoniana se presentó sintomatología dolorosa aún después de realizar el bloqueo anestésico. De acuerdo a nuestros resultados no concordamos con lo reportado por Malamed ${ }^{11}$ que describe que la técnica mentoniana puede alcanzar una anestesia pulpar profunda que permita realizar un tratamiento endodóntico.

\section{Conclusiones}

Se logró determinar que la técnica que cuenta con mayor eficacia para una anestesia profunda incluyendo el tejido pulpar es la técnica del bloqueo del nervio dentario inferior. La técnica del nervio incisivo no demostró una amplia eficacia para anestesiar el tejido pulpar.

\section{Bibliografía}

1. Dhindsa A, Pandit IK, Srivastava N, Gugnani N. Comparative evaluation of the effectiveness of electronic dental anesthesia with $2 \%$ lidocaine in various minor pediatric dental procedures: A clinical study. Contemp Clin Dent. 2011; 2(1): 27-30

2. Poorni S, Veniashok B, Senthilkumar AD, Indira R Ramachandran $S$ Anesthetic Efficacy of Four Percent Articaine for Pulpal Anesthesia by Using Inferior Alveolar Nerve Block and Buccal Infiltration Techniques in Patients with Irreversible Pulpitis: A Prospective Randomized Double-blind Clinical Trial. J Endod. 2011; 37(12): 1603-7.

3. Evidence-based review of clinical studies on local anesthetics. J Endod. 2009:1130-1134.

4. Palti Geller D, Almeida Machado C, Castro Rodrigues A, Oliveira Lima JE. Anesthetic technique for inferior alveolar nerve block: a new approach. J. Appl. Oral Sci. 2011; 19:11-5.

5. Balto K. Administration of Articaine Anesthesia may lead to Superior Profound Pulpal Anesthesia Compared with Lidocaine in Adult Patients. J Evid Based Dent Pract. 2011 Dec; 11(4):183-4.

6. Hass Da. An Update on Local Anesthetics in Denstristy. JADA 2002;68:546-51.

7. Isik K, Kalayci A, Durmus E. Comparison of depth of anesthesia in different parts of maxilla when only buccal anesthesia was done for maxillary teeth ex- traction. Int J Dent. 2011; 9: 1-3.

8. Pozos AJ, Martinez R, Aguirre P, Perez J. The effects of tramadol added to articaine on anesthesia duration. Oral Surg Oral Med Oral Pathol Oral Radio Endod. $2006 ; 102(5): 614-7$.

9. Foster W, Drum M, Reader A, Beck M. Anesthetic efficacy of buccal and lingual infiltrations of lidocaine following an inferior alveolar nerve block in mandibular posterior teeth. Anesth Prog. 2007; 54: 163-9.

10. Haas DA. Alternative mandibular nerve block techniques: a review of the Gow-Gates and AkinosiVazirani closed-mouth mandibular nerve block techniques. J Am Dent Assoc. 2011 Sep;142 Suppl 3:8S-12S

11. Malamed SF. Is the mandibular nerve block passé?. J Am Dent Assoc. 2011 Sep;142 Suppl 3:3S-7S

12. Stanley F. Malamed. Manual de Anestesia local. Elsevier. 2006. 249-253

13. Batista da Silva C, Berto LA, Volpato MC, Ramacciato JC, Motta RH, Ranali J, Groppo FC. Anesthetic efficacy of articaine and lidocaine for incisive/mental nerve block. J Endod. 2010; 36(3): 438-41.

14. Martínez MA. Anestesia bucal Guía práctica. Editorial médica panamericana. 2009. 110-113.

15. Hsiao-Wu GW, Susarla SM, White RR. Use of the cold test as a measure of pulpal anesthesia during endodontic therapy: a randomized, blinded, placebo-controlled clinical trial. J Endod. 2007;33(4):40610 . 